\title{
$t^{4}$ Report*
}

\section{Continuing Animal Tests on Cosmetic Ingredients for REACH in the EU}

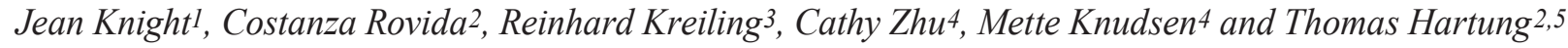 \\ ${ }^{1}$ White Rabbit Beauty LLC, Half Moon Bay, CA, USA; ${ }^{2}$ Center for Alternatives to Animal Testing Europe (CAAT-Europe), University of Konstanz, \\ Konstanz, Germany; ${ }^{3}$ Clariant Produkte (Deutschland) GmbH, Sulzbach, Germany; ${ }^{4}$ Knudsen \& CRC, Shanghai, China; ${ }^{5}$ Center for Alternatives to Animal \\ Testing (CAAT), Johns Hopkins University, Bloomberg School of Public Health, Baltimore, MD, USA
}

\begin{abstract}
EU cosmetic ingredients are governed by two regulations that conflict. Regulation EC 1223/2009, the Cosmetic Regulation, bans in vivo (animal) testing for cosmetic product safety assessments, including both final products and ingredients. At the same time, the Registration, Evaluation, Authorization and Restriction of Chemicals (REACH) regulation can impose in vivo testing of those same ingredients under its chemical testing requirements. Here, we examined REACH dossiers for chemicals for which the only reported use is cosmetics to determine the extent of new in vivo testing caused by REACH. We found the REACH database has 3,206 chemical dossiers with cosmetics as a reported use. Of these, 419 report cosmetics as the only use, and 63 of these have in vivo tests completed after the Cosmetic Regulation ban on in vivo testing. Registrants largely used alternative, non-animal methods to evaluate ingredients for REACH, but some still conducted new in vivo tests to comply with REACH requirements for toxicity data and worker safety assessments. In some cases, ECHA, the agency that evaluates REACH dossiers, rejected registrants' alternative methods as insufficient and required new in vivo tests. As ECHA continues to evaluate dossiers, more requests for in vivo tests are likely. REACH tests on cosmetic ingredients appear only as "industrial chemicals legislation" tests in EU reports. Given the importance to consumers and the cosmetic industry of having cosmetics free of animal testing, the public should be made aware of REACH testing until the conflict between the regulations is resolved.
\end{abstract}

\section{Introduction}

The use of in vivo tests for cosmetic products has raised ethical concerns for many years. Public opinion and the activity of animal welfare organizations induced the European Parliament in 2002 to enact the $7^{\text {th }}$ amendment to Directive 76/768/EEC on the safety of cosmetics, which introduced a phased ban on in vivo testing of cosmetic products and their ingredients (Hartung, 2008). The first phase, effective 2004, banned the sale of cosmetic products that had undergone in vivo testing. For cosmetic ingredients, the ban took effect in 2009 for in vivo tests for local health effects, such as eye irritation, and in 2013 for systemic effects, such as developmental effects. The ban deadlines are firm, irrespective of the availability of alternative non-animal tests (Adler et al., 2011).
This ban was confirmed in Regulation EC 1223/2009 (EC, 2009), which replaced Directive 76/768/EEC in 2009. Now, risk assessment of cosmetic ingredients in the EU must be performed based on historical in vivo studies, new in vitro (non-animal) studies, or other approaches not requiring new tests on vertebrate animals. Such approaches include the read-across approach, which predicts health effects of a chemical by using data from similar chemicals; the quantitative structure-activity relationship (QSAR) approach, which uses mathematical models to relate chemical structure to bioactivity; and the weight of evidence (WoE) approach, which uses data from multiple studies to develop conclusions (Patlewicz et al., 2014; Linkov et al., 2015; Chesnut et al., 2018; Rovida et al., 2020).

The Cosmetic Regulation allows in vivo tests for assessing cosmetic safety if the tests are performed for a non-cosmetic pur-

* a report of $t^{4}$ - the transatlantic think tank for toxicology, a collaboration of the toxicologically oriented chairs in Baltimore, Konstanz and Utrecht, sponsored by the Doerenkamp-Zbinden Foundation.

Received April 22, 2021; Accepted August 16, 2021; Epub August 18, 2021; @ The Authors, 2021

ALTEX 38(4), 653-668. doi:10.14573/altex.2104221

Correspondence:

Costanza Rovida, PhD

CAAT-Europe, University of Konstanz

Universitätstr. 10, 78464 Konstanz, Germany

(costanza.rovida@chimici.it)
This is an Open Access article distributed under the terms of the Creative Commons Attribution 4.0 International license (http://creativecommons.org/licenses/by/4.0/) which permits unrestricted use, distribution and reproduction in any medium, proviwhich permits unrestricted use, distribution 
pose, called a dual use. Most cosmetic ingredients have a dual use in other industries, such as in pharmaceuticals or as food/feed ingredients. Functional ingredients, such as surfactants, preservatives, and colorants, may have dual uses across many industries. Thus, the exemption for in vivo tests performed for a dual use limits the applicability of the Cosmetic Regulation ban.

In vivo tests conducted for other countries are not allowed for cosmetic safety assessments in the EU. Using such tests results in a marketing ban on the product. Some non-EU countries require in vivo data to authorize the marketing of cosmetic products. In the two largest cosmetic markets, the US and China, the US allows but does not require animal testing for cosmetic products and ingredients, except for some ingredients considered drugs; and China requires animal testing for both domestic and imported new cosmetic ingredients and special cosmetics. Recognizing the limitations of the EU ban, in 2018 the European Parliament adopted a resolution ${ }^{1}$ for a global end to animal testing for cosmetic products and their ingredients, demonstrating legislators' commitment to reinforce and expand the ban.

The regulation on Registration, Evaluation, Authorization and Restriction of Chemicals (REACH) was published in 2006 (Regulation EC 1907/2006). This regulation requires registration dossiers for all chemicals, including cosmetic ingredients, that are manufactured in or imported into the EU in a quantity above 1 ton/ year. The dossiers, which are submitted to the European Chemicals Agency (ECHA), include a full (eco)toxicological evaluation and a risk assessment relative to the declared use. The REACH dossiers are partially public and accessible in an open database ${ }^{2}$.

In the scope of REACH, the types of tests required for a chemical depend on the quantity of the chemical placed on the market, with requirements increasing across defined ranges: 1-10 tons/ year (Annex VII), 10-100 tons/year (Annex VIII), 100-1,000 tons/ year (Annex IX), and more than 1,000 tons/year (Annex X). The dossiers of substances registered according to Annex VIII onward must also contain a full chemical safety assessment (CSA) that addresses potential exposure scenarios. Cosmetic ingredients are exempted from a CSA for consumer exposure because the Cosmetic Regulation already requires this safety assessment. However, they are not exempted from a CSA for worker exposure during manufacture of the ingredient or the final cosmetic product.

REACH includes a mandate to use animal testing only as a last resort. Its Annex XI gives criteria for adapting the standard information requirements and waiving new in vivo tests (Hartung, 2010). It accepts the read-across, QSAR, and WoE approaches. Additionally, registrants are advised to use validated in vitro methods as soon as they are available. ECHA regularly publishes a report on the use of alternative methods in registration dossiers, with the latest published in $2020^{3}$.
Two important REACH amendments, Regulation EU 2016/863 and Regulation EU 2016/1688, were adopted in 2016. These require in vitro methods for the assessment, respectively, of skin/eye irritation and skin sensitization unless technically infeasible. This step was taken after the Organization for Economic Co-operation and Development (OECD) approved in vitro methods for skin irritation, eye irritation, and skin sensitization. It was a milestone to support the ban for cosmetic ingredients and direct toxicologists to always consider in vitro tests for chemical assessment.

Although important steps, REACH Annex XI and the 2016 amendments did not end other REACH requirements for new in vivo tests. As expected (Rovida and Hartung, 2009; Hartung and Rovida, 2009), the implementation of REACH increased the number of new in vivo tests on chemicals. It is too early to make a precise estimate, as many of those tests are ongoing and many others have been performed outside the EU (Busquet et al., 2020).

The relationship between REACH and the Cosmetic Regulation has been a matter of debate since the beginning. In 2014, ECHA published a factsheet titled "Interface between REACH and Cosmetics regulations", stating that a new in vivo test can be performed on a cosmetic ingredient to fulfil the REACH requirement for risk assessment for worker exposure and for ecosystem effects.

Many in the cosmetic industry and in animal welfare organizations have disagreed with this position. The debate culminated in 2020, when a cosmetic supplier protested before the ECHA board of appeal against an ECHA decision to require new in vivo tests for two cosmetic ingredients. In August 2020, the board of appeal upheld the ECHA decision ${ }^{5}$. The board of appeal decision led to an open letter to the EU institutions signed by animal welfare organizations and cosmetic companies, requesting a strict application of the ban on animal tests for cosmetic ingredients ${ }^{6}$.

In this ongoing discussion, a deeper understanding of the extent of continuing in vivo testing of cosmetic ingredients registered under REACH becomes important. The present paper identifies and analyzes the REACH dossiers of the chemicals declared to be used only for cosmetics to determine in vivo tests performed for REACH both overall and specifically after the cosmetic testing bans. Attention is also given to 2016, the year of publication of the two REACH amendments. We examine the use of in vivo and alternative methods for all human health endpoints and for one ecotoxicity endpoint, acute fish toxicity. Our aim is to provide regulators, industry, and consumers with a better understanding of the current situation of cosmetic ingredients testing under REACH to motivate a solution to the conflict between REACH and the Cosmetic Regulation. In keeping with this aim, we omit direct references to chemicals by name or

\footnotetext{
1 https://www.europarl.europa.eu/doceo/document/TA-8-2018-0202_EN.html

2 https://echa.europa.eu/information-on-chemicals/registered-substances (accessed 20.12.2020, 22.12.2020, 23.12.2020)

3 https://echa.europa.eu/documents/10162/0/alternatives_test_animals_2020_en.pdf/db66b8a3-00af-6856-ef96-5ccc5ae11026

4 https://echa.europa.eu/documents/10162/13628/reach_cosmetics_factsheet_en.pdf/2fbcf6bf-cc78-4a2c-83fa-43ca87cfb314

5 https://echa.europa.eu/documents/10162/5edc86c5-4397-54c6-831c-e53bcf90643d;

https://echa.europa.eu/documents/10162/23010712/a-010-2018_decision_en.pdf/46612b84-29af-29ea-9192-b2506f33c8ce

6 https://www.peta.org.uk/wp-content/uploads/2020/12/Open-Letter-Cosmetics-Animal-Testing-Ban-Effectively-Shredded.pdf
} 
EC/CAS number to prevent registrants from being blamed for a situation over which they have no control.

\section{Method}

We searched the ECHA database for substances for which the only reported use is cosmetics, extracted the test data for those substances, and organized the data in an Excel workbook for analysis.

\subsection{Determination of search parameters}

The ECHA search parameters used in the database search were:

- Product categories: PC 28, "perfumes, fragrances" and PC 39, "cosmetics, personal care products".

- Life cycles: "manufacture", "formulation", "uses at industrial sites" (production of final cosmetic), "consumer uses", and "widespread use by professional workers".

The product category options were based on the EU Cosmetic Regulation definition of a cosmetic product: “.... any substance or mixture intended to be placed in contact with the external parts of the human body (epidermis, hair system, nails, lips and external genital organs) or with the teeth and the mucous membranes of the oral cavity with a view exclusively or mainly to cleaning them, perfuming them, changing their appearance, protecting them, keeping them in good condition or correcting body odours" (Article 2) (EC, 2009).

The life cycle options, which relate to the life stages of a chemical from its manufacture through to its end use, were based on the Article 2 definitions for "substance" and "end user" (EC, 2009). A "substance" is "a chemical element and its compounds in the natural state or obtained by any manufacturing process". An "end user" is "either a consumer or professional using the cosmetic product".

Going forward, this paper uses the term "cosmetic-only substance" to mean an ingredient for a cosmetic product used by consumers or professionals and used in no other industry at any stage from manufacture through end use. This paper uses the term "substance" rather than "ingredient" to be consistent with the usage in the Cosmetic and REACH regulations.

\subsection{Identification of cosmetic-only substances in the ECHA database}

A series of ECHA database searches ${ }^{7}$, performed in December 2020, identified cosmetic-only substances registered in the ECHA database:

1. Searched for all cosmetic substances registered in the ECHA database: The search parameters were product category: PC 28, PC 39; life cycles: none selected; search operator: OR. The search identified 3,206 substances with PC 28 or PC 39 reported for any cosmetic substance life cycle.

2. Removed cosmetic substances with other reported product categories: The search parameters were product category: all selected except PC 28 and PC 39; life cycles: none selected; search operator: OR. This search identified all substances with other, non-cosmetic uses for any life cycle. Note that we could have selected the applicable life cycles instead of leaving them blank, but leaving them blank accomplished the same thing more efficiently. Excel functions were then used to compare the search results with the list of cosmetic substances from step 1. This identified cosmetic substances with other product categories besides cosmetics, and those substances were removed from the list of cosmetic substances, leaving 469.

3. Identified those with industrial life cycle use for further inspection: The search parameter was life cycles: uses at industrial sites. This search identified all REACH substances with an industrial use. Excel functions were used to compare these substances to the list of cosmetic substances from step 2, identifying cosmetic substances with industrial use. These required further inspection, because dossiers often do not include PC categories for the industrial use life cycle. The dossiers were reviewed to determine the industrial uses, and substances with non-cosmetic industrial uses or unclear data were removed.

4. Removed substances with non-active status and intermediates with no data: Substances with a status of "cease manufacture" or "no longer valid" and substances registered as an "intermediate" type with no toxicity data were removed from the list of cosmetic substances.

The final result of these searches was a list of 419 dossiers for 413 unique cosmetic-only substances.

Note that searches cannot find information entered as general text; for example, product categories are sometimes entered as text (e.g., "end use as cosmetics") rather than as a PC category. Likely some dossiers of cosmetic ingredients were not found. Also, the ECHA database is frequently updated with new and revised dossiers, and new searches may have slightly different results than this December 2020 search. Our search was performed before Brexit, and dossiers that had only UK registrants are now marked as "no longer valid" in the ECHA database.

\subsection{Identification of INCI names for the substances}

The cosmetic industry has its own names for cosmetic ingredients, called International Nomenclature of Cosmetic Ingredients names, or INCI names. They are assigned by the International Nomenclature Committee and published by the Personal Care Products Council ${ }^{8}$. Most but not all cosmetic ingredients have INCI names.

INCI names were retrieved from the ECHA dossiers, if included. If not, the INCI names were obtained from the EU's Cosmetic Ingredient database, CosIng 9 . The CosIng database was downloaded, and Excel functions were used to match the EC or CAS numbers in CosIng to those in the ECHA dossiers of the cosmetic substances, returning the INCI name and cosmetic function if there was a match. For substances with no EC or CAS number,

\footnotetext{
7 https://echa.europa.eu/information-on-chemicals/registered-substances (accessed 20.12.2020, 22.12.2020, 23.12.2020)

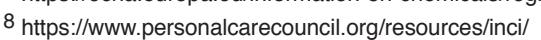

9 https://ec.europa.eu/growth/sectors/cosmetics/cosing_en (accessed 20.01.2021). The "Inventory of Ingredients" option provides a download link on the results page.
} 
Tab. 1: Summary of the numbers of dossiers for cosmetic ingredients in the ECHA database (https://echa.europa.eu/ information-on-chemicals/registered-substances), accessed December 20-23, 2020

In rare cases, one substance may have two dossiers, thus the $419 \mathrm{REACH}$ dossiers correspond to 413 unique cosmetic-only substances. The identification of the cosmetic-only ingredients eliminated any substance for which there was a suspicion of dual use. This means that the data presented here could be underestimating the number of cosmetic-only ingredients that have been registered under REACH. Regarding the in vivo studies performed after the ban of the Cosmetic Regulation, the month of March was used as a reference.

\begin{tabular}{|l|l|}
\hline REACH registration dossiers & Number of dossiers \\
\hline With a cosmetic use & 3,206 \\
\hline With cosmetic as only use & 419 \\
\hline With alternative methods only & 82 \\
\hline With in vivo studies performed after 2009 & 121 \\
\hline With in vivo studies performed after the cosmetic ban in March 2009 or 2013, depending on health endpoint & 63 \\
\hline
\end{tabular}

the dossier was searched for a trade name or other identifier that could be used to find the INCI name.

Plant extracts from the same plant (e.g., leaf extract, flower extract) have individual INCI names but have the same EC number. To find the correct INCI name for these substances, their ECHA dossiers were reviewed to determine the registered extract.

\subsection{Verification of cosmetic-only substances}

$\mathrm{REACH}$ registration dossiers for the cosmetic-only substances were manually checked to confirm that PC 28 or PC 39 were the only product categories reported for any life cycle use. Dossiers were also reviewed for information that corroborates cosmetics as the sole use. This information includes:

- Explicit statements that a substance is used only for cosmetics.

- Use of a trade name for the substance, and the trade name is that of a cosmetic ingredient.

- Use of the INCI name for the substance.

- Registrants who are cosmetic companies or cosmetic industry suppliers.

Substances with in vivo toxicity tests conducted after the Cosmetic Regulation deadlines (11 March 2009 and 11 March 2013) were flagged for additional verification of cosmetic-only status. Registrants with multiple substances on the list were contacted directly. For substances not confirmed by registrants, verification involved checking registrant websites for potential dual uses exempted from REACH registration (i.e., pharmaceuticals, food/ feed additives, biocides, and plant protection products); checking records of the European Food Safety Authority (EFSA) and European Medicines Agency (EMA) for opinions on the substances, indicating a dual use; and reviewing dual-use information in safety assessments from the EU Scientific Committee on Consumer Safety (SCCS) and the Cosmetic Ingredient Review (CIR) panel in the US.

\subsection{Extraction of dossier data for analysis}

Data were collected by opening each dossier directly from the ECHA database and manually reviewing the dossier. An automated process was not possible because ECHA dossiers have no standard format for toxicity data, and each registrant may enter the data in different ways. This is particularly an issue for read- across studies, which sometimes are not labeled as read-across and require careful reading to determine if they are experimental studies on the registered substance or read-across studies using a similar substance.

Manual review was also the only way to obtain contextual information that could help identify the purpose of a test (e.g., for another country's regulation) and the rationale for approaches (e.g., if an in vitro test was not possible for technical reasons, this triggered an in vivo approach). To reduce human errors and maintain consistency across the collected data, one author collected all the data and another author reviewed it.

The following data were collected and entered in an Excel workbook:

- General information: EC number, CAS number, EC name, tonnage range, constituent type (mono-constituent, multi-constituent, or UVCB (unknown or variable composition, complex reaction product, or biological material)), first publication date, last update, and registrants.

- Toxicity study information: Health endpoint, study type, test method (application if QSAR), utility of study (key, supporting, weight of evidence), reliability of study (1-4), and use of read-across. If a study was read-across, additional information was noted, i.e., read-across type (category or structural ana$\log$ ), read-across utility, and read-across reliability.

- Study dates: Study dates were used to identify toxicity studies conducted after regulatory deadlines. REACH dossiers include the following fields for reporting dates: "study period" for the study itself and "year" and "report date" for the data source. For study year, the "study period" year was entered if the information was reported. If that field was blank, the "year" field was used if it could be corroborated by information in the study itself, for example, in-life dates. Studies with in-life dates that straddled a deadline were considered to be after the deadline, because the owner would have known that the test would extend past the deadline. If the study year was unclear, it was recorded as ND for "no date". The report year was also recorded, using the year in the "report date" field. If that field was blank, the report date was entered as ND.

Excel functions and formulas were then used to extract, summarize, and chart the data. 
Tab. 2: Number of REACH registration dossiers and endpoint records per tonnage range

For each dossier, the endpoints with data were counted. This is not the number of tests that were analyzed, as one substance may have several studies for the definition of the endpoint. In the REACH registration dossiers, an endpoint is not included if it is not requested by the REACH Annexes for the registration, if there is no public justification for waiving, or if the registrant entered it into the confidential area of the dossier and it is therefore not accessible in the ECHA database. The analysis was performed in December 2020.

\begin{tabular}{|c|c|c|c|c|c|c|}
\hline & $\begin{array}{l}\text { Annex VII } \\
1-10 \text { tons/yr }\end{array}$ & $\begin{array}{l}\text { Annex VIII } \\
10-100 \text { tons/yr }\end{array}$ & $\begin{array}{l}\text { Annex IX } \\
100-1,000 \text { tons/yr }\end{array}$ & $\begin{array}{l}\text { Annex } X \\
>1,000 \text { tons/yr }\end{array}$ & $\begin{array}{l}\text { Confidential } \\
\text { tons/yr }\end{array}$ & Total \\
\hline Number of dossiers & 233 & 124 & 48 & 11 & 3 & 419 \\
\hline Number of unique substances ${ }^{a}$ & & & & & & 413 \\
\hline \multicolumn{7}{|l|}{$\begin{array}{l}\text { Number of dossiers with endpoint } \\
\text { data, including waivers }\end{array}$} \\
\hline Skin irritation & 220 & 123 & 48 & 11 & 3 & 405 \\
\hline Eye irritation & 219 & 123 & 48 & 11 & 3 & 404 \\
\hline Skin sensitization & 221 & 123 & 48 & 11 & 3 & 406 \\
\hline Genetic toxicity & 217 & 123 & 48 & 11 & 3 & 402 \\
\hline Acute toxicity - oral & 218 & 123 & 48 & 11 & 3 & 403 \\
\hline Acute toxicity - dermal & 43 & 94 & 48 & 11 & 2 & 198 \\
\hline Acute toxicity - inhalation & 19 & 89 & 46 & 11 & 2 & 167 \\
\hline Repeated dose toxicity - oral & 38 & 96 & 46 & 11 & 2 & 193 \\
\hline Repeated dose toxicity - dermal & 8 & 46 & 29 & 10 & 2 & 95 \\
\hline Repeated dose toxicity - inhalation & 8 & 42 & 24 & 8 & 1 & 83 \\
\hline Reproductive toxicity & 17 & 91 & 48 & 11 & 2 & 169 \\
\hline Developmental toxicity & 27 & 55 & 42 & 10 & 2 & 136 \\
\hline Toxicokinetics & 36 & 88 & 45 & 10 & 2 & 181 \\
\hline Acute fish toxicity & 45 & 97 & 47 & 11 & 2 & 202 \\
\hline
\end{tabular}

a The total number of unique substances is 413 , because two substances have three dossiers each, and two substances have two dossiers each. Note that when substances have more than one co-registrant, the tonnage band reported in the public database may not correspond to the tonnage band of the registered substance. ECHA may consolidate the ranges into one range.

\section{Cosmetic-only substances in the REACH database}

The ECHA database search (December 2020) identified 419 REACH dossiers for 413 unique cosmetic-only substances. The substance characteristics and toxicity studies are reviewed here, with the key data summarized in Table 1. Note that our analysis eliminated any substance for which there was a suspicion of dual use. This means that the data presented here could be underestimating the number of cosmetic-only ingredients that have been registered under REACH.

\subsection{Substance characteristics}

Most cosmetic-only substances in REACH have low production volumes, which is typical for cosmetic ingredients. Of the 419 dossiers, 233 (55\%) are for substances with volumes of 1-10 tons/year (REACH Annex VII) and 124 (30\%) are for substances with volumes of 10-100 tons/year (REACH Annex VIII). The remaining $15 \%$ of dossiers comprise 48 substances $(11 \%)$ in the 100-1,000 tons/year range (Annex IX), 11 substances $(3 \%)$ in the $1,000+$ tons/year range (Annex X), and 3 substances (1\%) with confidential tonnage. Note that the ton- nage band of the substances retrieved from the ECHA database may not be identical with that provided in the original dossiers submitted by the registrants.

The substance types are $46 \%$ mono-constituent, $41 \%$ UVCB, $12 \%$ multi-constituent, and $1 \%$ not reported.

\section{Cosmetic uses}

INCI cosmetic functions for 324 of the cosmetic-only substances were obtained from the CosIng database ${ }^{9}$. The substances cover more than 45 cosmetic functions, with the most prevalent being skin conditioning, surfactant, emollient, hair conditioning, hair dye, antistatic, emulsifying, and perfume/fragrance. About $10 \%$ of the substances, mainly hair dyes, have a restricted use under Annex III of the Cosmetic Regulation. Another $8 \%$ are colorants, preservatives, and UV filters allowed under Cosmetic Regulation Annexes IV-VI, some with concentration or use restrictions.

Note that REACH has specific rules for naming UVCBs and multi-constituent substances. Consequently, some cosmetic substances are registered in REACH with new names and EC list numbers, losing the connection with INCI data. We were unable to determine INCI functions for 89 of the 413 substances. 
Tab. 3: Study types in REACH registration dossiers by relative percentage of endpoint records

The table gives the relative percentage of study type for that endpoint, calculated as (total count for that study type)/(total count for all study types for that endpoint). This is the table equivalent of a $100 \%$ bar chart. The table counts whether a particular study type or approach is used for the endpoint, not the number of those studies. For example, if a dossier has 3 in vivo tests for an endpoint, it gets just one count in the in vivo column for that endpoint. Note that dossiers may have multiple study types for an endpoint, e.g., for the eye irritation endpoint, a dossier may have both in vitro studies and QSAR studies, and so have one count in each column. In particular, the WoE approach must have more than one test, by definition. The analysis was done by manually opening each dossier and analyzing the content as it appears in the ECHA database.

\begin{tabular}{|c|c|c|c|c|c|c|c|c|}
\hline Endpoint & $\begin{array}{l}\text { In vivo } \\
\text { study }^{\mathrm{a}}\end{array}$ & $\begin{array}{l}\text { In vitro } \\
\text { study }\end{array}$ & $\begin{array}{l}\text { Human } \\
\text { study }\end{array}$ & $\begin{array}{l}\text { QSAR/ other } \\
\text { calculation }\end{array}$ & $\begin{array}{l}\text { Expert } \\
\text { statement }\end{array}$ & $\begin{array}{l}\text { Read- } \\
\text { across }\end{array}$ & WoE & Waiver \\
\hline Skin irritation & $41.2 \%$ & $29.3 \%$ & $5.6 \%$ & $1.9 \%$ & $0.2 \%$ & $15.5 \%$ & $6.1 \%$ & $0.2 \%$ \\
\hline Eye irritation & $38.6 \%$ & $32.3 \%$ & $0.2 \%$ & $1.9 \%$ & $0.4 \%$ & $16.3 \%$ & $8.9 \%$ & $1.5 \%$ \\
\hline Skin sensitization & $41.2 \%$ & $13.5 \%$ & $5.9 \%$ & $4.5 \%$ & $1.1 \%$ & $18.4 \%$ & $13.9 \%$ & $1.6 \%$ \\
\hline Genetic toxicity & $11.2 \%$ & $59.1 \%$ & $0.0 \%$ & $2.2 \%$ & $1.7 \%$ & $18.4 \%$ & $7.2 \%$ & $0.2 \%$ \\
\hline Acute toxicity - oral & $55.0 \%$ & $2.6 \%$ & $0.0 \%$ & $3.2 \%$ & $1.0 \%$ & $24.6 \%$ & $12.3 \%$ & $1.4 \%$ \\
\hline Acute toxicity - dermal & $38.4 \%$ & $0.4 \%$ & $0.0 \%$ & $4.4 \%$ & $0.9 \%$ & $23.6 \%$ & $10.9 \%$ & $21.4 \%$ \\
\hline Acute toxicity - inhalation & $8.4 \%$ & $0.6 \%$ & $0.0 \%$ & $4.5 \%$ & $0.0 \%$ & $8.4 \%$ & $5.6 \%$ & $72.6 \%$ \\
\hline Repeated dose toxicity - oral ${ }^{b}$ & $52.3 \%$ & $0.0 \%$ & $0.0 \%$ & $0.4 \%$ & $0.4 \%$ & $34.0 \%$ & $10.2 \%$ & $2.6 \%$ \\
\hline Repeated dose toxicity - dermal & $18.0 \%$ & $0.0 \%$ & $0.0 \%$ & $1.0 \%$ & $0.0 \%$ & $23.0 \%$ & $5.0 \%$ & $53.0 \%$ \\
\hline Repeated dose toxicity - inhalation & $7.3 \%$ & $0.0 \%$ & $0.0 \%$ & $0.0 \%$ & $0.0 \%$ & $13.4 \%$ & $0.0 \%$ & $79.3 \%$ \\
\hline Reproductive toxicity ${ }^{b}$ & $32.1 \%$ & $1.0 \%$ & $0.0 \%$ & $1.0 \%$ & $1.4 \%$ & $36.4 \%$ & $11.5 \%$ & $16.7 \%$ \\
\hline Developmental toxicity ${ }^{b}$ & $48.1 \%$ & $0.6 \%$ & $0.0 \%$ & $0.0 \%$ & $0.0 \%$ & $39.4 \%$ & $7.5 \%$ & $4.4 \%$ \\
\hline Toxicokinetics & $18.3 \%$ & $12.7 \%$ & $2.8 \%$ & $5.6 \%$ & $31.7 \%$ & $16.7 \%$ & $8.7 \%$ & $3.6 \%$ \\
\hline Acute fish toxicity & $51.4 \%$ & $7.2 \%$ & $0.0 \%$ & $7.2 \%$ & $0.4 \%$ & $22.3 \%$ & $9.2 \%$ & $2.4 \%$ \\
\hline
\end{tabular}

a This is for in vivo studies conducted on the registered cosmetic ingredient. In vivo studies conducted on related substances in a readacross approach are considered a read-across study type. ${ }^{b}$ Many registrants use a single test method, OECD TG 422 , for all three endpoints; similarly, the read-across studies may use the same OECD TG 422 study for all three endpoints. In this table, studies used for more than one endpoint are included for each endpoint for which they are used.

Cosmetic substance life cycles

The ECHA life cycles reported in the cosmetic-only dossiers were:

- Manufacture: 238 substances; typically relates to manufacture of the substance itself, but sometimes designates final cosmetic production

- Formulation or re-packing: 354 substances; examples are formulation of the substance into a mixture to create other cosmetic ingredients or to create a cosmetic prototype or product

- Uses at industrial sites: 48 substances; typically relates to production of the final cosmetic product

- Consumer use: 381 substances

- Widespread uses by professional workers: 160 substances

The first three listed life cycles relate to production of cosmetics and may involve worker exposure. A total of $362(86 \%)$ of the cosmetic-only dossiers report one or more of these three production life cycles. This has implications for future animal testing, given ECHA's decision to allow animal tests on cosmetic-only substances if workers' jobs may expose them directly or indirectly to the substance ${ }^{4}$.

\section{"Dual use" cosmetic substances}

This paper focuses on the 419 dossiers for 413 cosmetic-only substances, but the ECHA database search also returned more than 2700 other dossiers with cosmetics reported as one of multiple uses. The reported other product categories (PCs) for the cosmetic substances include the full range of possible categories, from PC 0 to PC 42; however, the most common additional categories are household products: washing and cleaning products (PC 35); polishes and wax blends (PC 31); biocides (PC 8), which are disinfectants and pest control products; and air care products (PC 3). The median number of other uses was 6 , and the most common number of other uses was 4 . It is beyond the scope of this paper to examine tests on these dual-use ingredients, but given that most cosmetic ingredients have a dual use, this use scenario should be part of any discussion on cosmetic testing.

\subsection{Toxicity studies}

All but 12 of the 419 cosmetic-only dossiers have some toxicity data. Most dossiers, $95 \%$, have data for at least the following endpoints: eye irritation, skin irritation, skin sensitization, acute oral toxicity, and genetic toxicity (Tab. 2). 
Tab. 4: Use of alternative methods in REACH registration dossiers

Data on the distribution of the approaches are reported in Table 3. Eighty-two dossiers contain no in vivo studies and fully rely on alternative methods for all human health and ecotoxicity endpoints considered in the dossier.

\begin{tabular}{|c|c|c|c|}
\hline Endpoint & $\begin{array}{l}\text { Total no. of dossiers with } \\
\text { endpoint data, excluding } \\
\text { waivers }\end{array}$ & $\begin{array}{l}\text { No. of dossiers with only } \\
\text { alternative methods for } \\
\text { endpoint }^{\mathrm{a}}\end{array}$ & $\begin{array}{l}\% \text { dossiers with only } \\
\text { alternative methods for } \\
\text { endpoint }^{b}\end{array}$ \\
\hline Skin irritation & 404 & 185 & $46 \%$ \\
\hline Eye irritation & 396 & 187 & $47 \%$ \\
\hline Skin sensitization & 397 & 161 & $41 \%$ \\
\hline Genetic toxicity & 401 & 333 & $83 \%$ \\
\hline Acute toxicity - oral & 396 & 115 & $29 \%$ \\
\hline Acute toxicity - dermal & 149 & 59 & $40 \%$ \\
\hline Acute toxicity - inhalation & 37 & 21 & $57 \%$ \\
\hline Repeated dose toxicity - oral & 187 & 62 & $33 \%$ \\
\hline Repeated dose toxicity - dermal & 42 & 24 & $57 \%$ \\
\hline Repeated dose toxicity - inhalation & 18 & 12 & $67 \%$ \\
\hline Reproductive toxicity & 134 & 67 & $50 \%$ \\
\hline Developmental toxicity & 129 & 51 & $40 \%$ \\
\hline Toxicokinetics & 172 & 133 & $77 \%$ \\
\hline Acute fish toxicity & 196 & 62 & $32 \%$ \\
\hline
\end{tabular}

a Alternative methods were in vitro/ex vivo studies, read-across studies, QSARs or other calculations, expert statements, or studies with human volunteers. ${ }^{b}$ Calculated as (Column 4: No. of dossiers with only alternative methods for that endpoint)/(Column 2: Total no. of dossiers with data for that endpoint). It excludes dossiers that used a waiver because a waiver is not endpoint data. ${ }^{\mathrm{C}}$ Excluding ecotoxicity endpoints, the number of dossiers using alternative methods for all endpoints is 89 .

Many dossiers, especially in tonnage ranges above 10 tons/ year, have data on additional endpoints: $36 \%$ of Annex VII substances (1-10 tons/year), 84\% of Annex VIII substances (10100 tons/year), and all dossiers for substances in Annex IX (1001,000 tons/year) and Annex X (> 1,000 tons/year). This is expected because REACH requires additional endpoint evaluations for higher tonnage ranges.

\section{Overview of study types}

In vivo tests pre-dating REACH are the most common study type for most endpoints (Tab. 3). The exceptions are genetic toxicity, reproductive toxicity, and toxicokinetics. For genetic toxicity, in vitro tests dominate; for reproductive toxicity, the read-across approach is more common; and for toxicokinetics, the main study type is an expert statement based on available data (Tab. 3 ). Waivers are the most common approach for acute and repeated-dose inhalation toxicity and repeated-dose dermal toxicity.

Next to genetic toxicity ( $59 \%$ of dossiers), in vitro studies are well represented for skin irritation (29\% of dossiers) and eye irritation (32\% of dossiers), reflecting the availability of in vitro methods for these endpoints. The main study type for these latter endpoints is historical in vivo studies, and the in vitro tests are more recent.
The read-across approach, sometimes combined with the weight-of-evidence approach, appears in $16-25 \%$ of the dossiers for the endpoints skin and eye irritation, skin sensitization, acute oral toxicity, and genetic toxicity (Tab. 3).

The read-across approach has most use for the repeated-dose endpoints: $34-39 \%$ of dossiers use this approach for repeated-dose oral toxicity, reproductive toxicity, and developmental toxicity.

QSARs have limited use, found mainly for acute fish toxicity ( $7 \%$ of dossiers with this endpoint) and toxicokinetics $(6 \%$ of dossiers with this endpoint). Weight-of-evidence also has limited use, with $0-14 \%$ of dossiers including this method for an endpoint. Skin sensitization has the most use, at $14 \%$, followed by acute oral and dermal toxicity, repeated-dose oral toxicity, and reproductive toxicity at $10-12 \%$ of dossiers with those endpoints.

\section{Use of alternative approaches}

Eighty-two dossiers (20\% of the 419 analyzed) contain no in vivo studies and fully rely on alternative methods for all human health and ecotoxicity endpoints considered in the dossier (Tab. 4). Excluding ecotoxicity endpoints, the number is 89 .

Many other dossiers use exclusively alternative methods for at least some endpoints (Tab. 4). Acute oral toxicity has the least percentage use of exclusively alternative methods, reported 

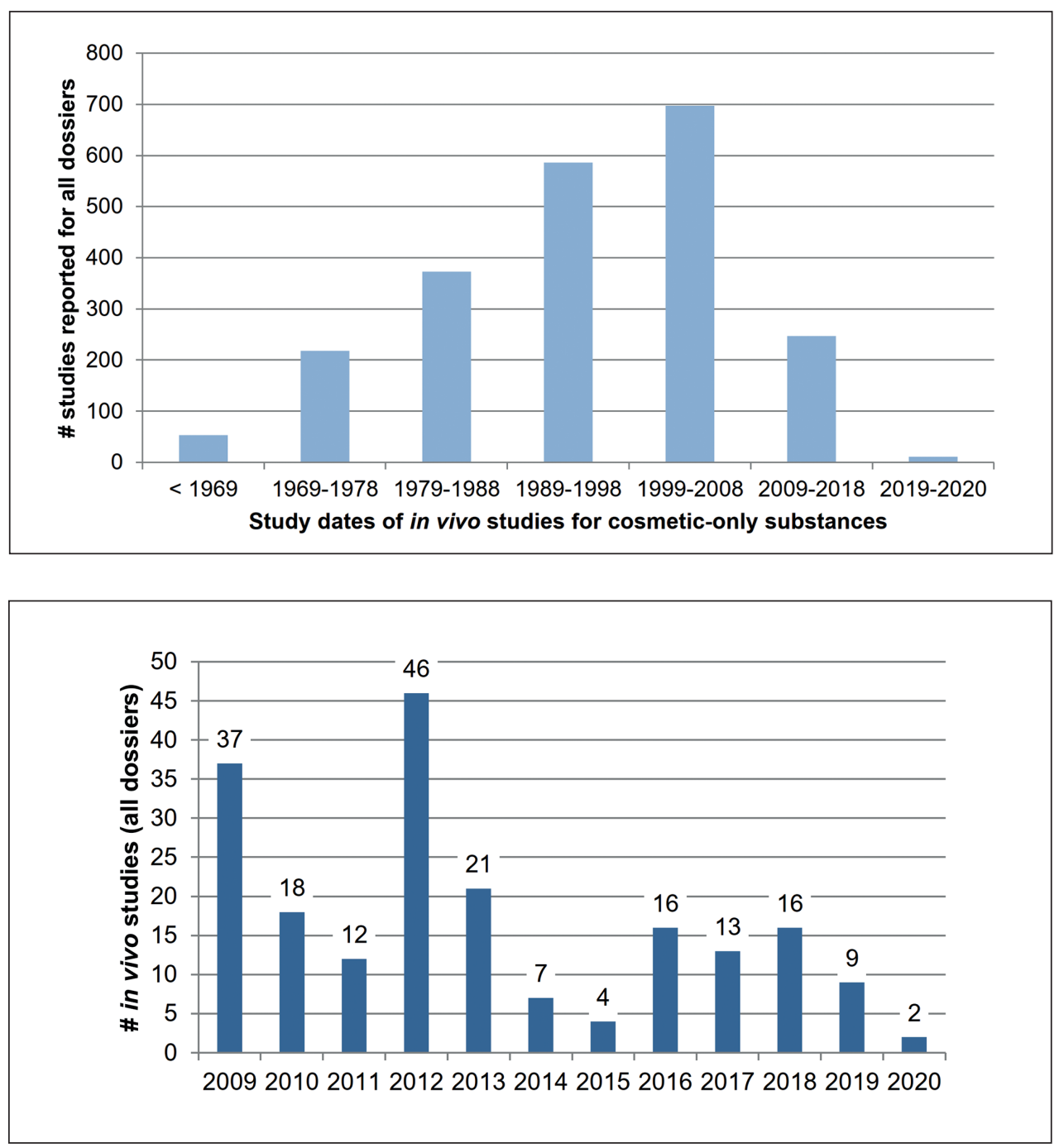

Fig. 1: All in vivo studies reported in cosmetic-only dossiers by study or report date as indicated in the ECHA database

Includes studies reported in publications; most of these pre-date 2009. The interval was selected to be consistent with the period when $\mathrm{REACH}$ was implemented in the EU. REACH was published in 2006 and entered into force in 2008. Companies started implementing it in 2009 , and the first registration deadline was in 2010.

Fig. 2: Number of unique in vivo tests for cosmetic-only substances in 2009-2020 extracted from the ECHA database

The total number shown, 201, excludes 16 studies reported in dossiers or by registrants as being for a non-REACH purpose, indicating either a dual use or compliance with a non-EU country. in $29 \%$ of dossiers with data for that endpoint. The most common alternative method for acute oral toxicity is read-across (25\%, Tab. 3). Only thirteen (3\%, Tab. 3) of the 419 dossiers include the in vitro NRU assay for acute oral toxicity, usually in a weight-of-evidence approach with historical in vivo studies, read-across studies, and QSAR studies. The low number may be due to the availability of historical data and use of read-across to avoid new animal testing.

\section{Use of in vivo studies}

ECHA uses 2009 as the cutoff year that defines "old" studies, stating "2009 is taken as a significant point in time, as it defined the studies that generally should be conducted and motivated by the REACH requirements - new studies that had to be done by registrants to ensure the safe use and no viable alternative was available" (ECHA, 2020).

About $88 \%$ of the 2,180 in vivo tests for the cosmetic-only substances are older studies, pre-dating 2009 (Fig. 1). Most of these date to 1975-2008, although some date even to the 1930 s.
This reflects the REACH requirement for registrants to enter all available studies they can find for a substance. This has created a substantial repository of historical toxicity studies for registered substances and allows us to view trends over time. Figure 1 shows the total in vivo studies on the substances in the 419 cosmetic-only dossiers. It shows a significant decline of in vivo tests for cosmetic-only substances after 2009, coinciding with the implementation of the ban of the Cosmetic Regulation on animal testing.

The trends for in vivo studies for individual health endpoints mirror the 2009 decline shown in Figure 1; however, the size of the drop differs and is largely related to the availability of in $v i$ tro methods for the endpoint. Genetic toxicity and eye and skin irritation, for which in vitro methods are available, drop by about $90 \%$. The least decline occurred for endpoints with no validated alternative methods: acute toxicity $(-51 \%)$, repeated-dose toxicity $(-51 \%)$, and reproductive toxicity $(+4 \%)$. Although developmental toxicity also has no available alternatives, it shows a greater decline $(-60 \%)$ because $\mathrm{REACH}$ requires developmental 
Tab. 5: In vivo tests done after Cosmetic Regulation deadlines

Summary of the in vivo studies performed after 2009, with details on which were recorded after the ban introduced by the Cosmetic Regulation. Acute fish toxicity, which has 26 in vivo studies after 11 March 2009, is excluded because the Cosmetic Regulation does not include ecotoxicity, so there is no applicable deadline.

\begin{tabular}{|c|c|c|c|c|c|}
\hline & Tests since $2009^{a}$ & $\begin{array}{l}\text { Tests after } \\
\text { cosmetic ban }\end{array}$ & $\begin{array}{l}\text { REACH } \\
\text { confirmed/likely }\end{array}$ & $\begin{array}{l}\text { non-REACH } \\
\text { confirmed }^{C}\end{array}$ & $\begin{array}{l}\text { OECD Test } \\
\text { Methods }^{d}\end{array}$ \\
\hline \multicolumn{6}{|l|}{ Cosmetic ban = 11 Mar 2009} \\
\hline Skin irritation & 10 & 8 & 6 & 2 & 404 \\
\hline Eye irritation & 9 & 9 & 8 & 1 & 405 \\
\hline Genetic toxicity & 3 & 2 & 1 & 1 & 474 \\
\hline Acute toxicity - oral & 44 & 41 & 36 & 5 & $423(31) ; 420(5)$ \\
\hline Acute toxicity - dermal & 7 & 7 & 5 & 2 & 402 \\
\hline \multicolumn{6}{|l|}{ Cosmetic ban = 11 Mar 2013} \\
\hline Skin sensitization & 44 & 16 & 15 & 1 & $429(13), 406(2)$ \\
\hline Repeated dose toxicity - oral $\left.\right|^{e}$ & 37 & 9 & 7 & 2 & $\begin{array}{l}407(3), 408(1) \\
422(3)\end{array}$ \\
\hline Reproductive toxicity & 20 & 8 & 7 & 1 & $421(6), 443(1)$ \\
\hline Developmental toxicity & 13 & 3 & 3 & 0 & 414 \\
\hline Toxicokinetics & 4 & 1 & 0 & 1 & None \\
\hline TOTAL & 191 & 104 & 88 & 16 & \\
\hline
\end{tabular}

a Some in vivo tests were conducted before the Cosmetic Regulation deadlines. That is why the number of tests since 2009 exceeds the number of tests after the Cosmetic Regulation deadline for some endpoints. ${ }^{\mathrm{b}}$ Test is confirmed or likely to have been done for REACH. "Confirmed" means this was confirmed directly by the registrant. "Likely" means the dossier information indicates the test was probably performed for REACH, based on test dates, test approaches, and dossier statements. ${ }^{\mathrm{C}}$ Test was done for a purpose other than REACH, confirmed either by the registrant or in the dossier. ${ }^{d}$ For the "REACH confirmed/likely" tests. ${ }^{e}$ Includes OECD 422 studies, which are used for both repeated dose-oral and reproductive toxicity. Here they are included only under repeated dose-oral toxicity to avoid duplicates.

toxicity only for substances with tonnages above 100 tons/year, and most cosmetic substances have tonnages below this.

\section{Newer in vivo tests}

Newer in vivo tests (2009-2020) are reported in 121 dossiers (29\%). Combined, they include a total of 201 tests during that period, excluding tests known to be done for a different purpose. The pattern of tests generally reflects the REACH deadlines (Fig. 2), with most tests occurring in the years immediately before or of the three REACH deadlines for registering existing substances, i.e., 2010 for substances in Annex X (> 1,000 tons/year), 2013 for substances in Annex IX (100-1,000 tons/year), and 2018 for substances in Annexes VIII (10-100 tons/year) and VII (1-10 tons/year).

\section{Ecotoxicity tests}

After 2009, 26 fish toxicity tests were performed for 22 cosmetic-only substances. Of these substances, 6 also underwent new in vivo studies after 2009 for human health endpoints, and the remaining 16 did not. The cosmetic ban does not apply to in vivo studies for ecotoxicity, and these tests are not further considered in the paper; however, we note that fish are vertebrate animals that suffer during toxicity experiments and deserve care to avoid new tests.

\section{In vivo tests done after the Cosmetic Regulation deadlines}

The Cosmetic Regulation ban on in vivo testing took effect on 11 March 2009 for skin and eye irritation, acute toxicity, and genetic toxicity, and on 11 March 2013 for all other human health endpoints. Sixty-three cosmetic-only substances had in vivo tests for human health endpoints after the Cosmetic Regulation deadlines for in vivo tests for the respective endpoints, making a total of 104 tests (Tab. 5). These substances are analyzed further here.

\subsection{Substance characteristics}

The 63 substances are made up of $65 \%$ Annex VII $(<10$ tons/ year), 24\% Annex VIII (10-100 tons/year), 8\% Annex IX (1001,000 tons/year), and 3\% Annex X and confidential tonnage (1 substance each). The proportion of Annex VII substances is slightly higher than in the total set of 413 substances, which is made up of 55\% Annex VII, 30\% Annex VIII, 11\% Annex IX, 3\% Annex X, and $1 \%$ confidential tonnage.

Two substances are restricted under Annex III of the Cosmetic Regulation. Another five substances are colorants, preservatives, or UV filters allowed under Annexes IV-VI, some with concentration or use restrictions. 
Two substances of the 63 substances have SCCS opinions, including one of the substances restricted under Annex III. One opinion concludes the substance is safe at the current concentration limit; and one opinion, addressing sensitization only, concludes that there is allergenic potential but that the risk of sensitization is low.

The ECHA database includes a section on the classification of the substances according to Classification, Labeling, and Packaging (CLP) rules. Many of these 63 substances are not classified for any hazard, and none have a classification as carcinogenic, mutagenic, or toxic for reproduction.

\subsection{Verification that REACH is test purpose}

Seven registrants were contacted to confirm that substances they registered of the set of 63 substances were tested for REACH. Three responded, representing 15 of the substances and 27 of the tests. Dossiers for the other substances were reviewed for information indicating whether tests were done for REACH or another purpose. The information obtained on all 63 substances is as follows:

- 13 substances were directly confirmed by the registrants (12 substances) or in the dossier (1 substance) to have been tested for REACH.

- 42 substances were likely tested for REACH, based on dossier statements, test dates near REACH registration deadlines, and test approaches that match REACH guidance, such as use of $i n$ vitro methods first.

- 1 substance had one test likely done for REACH and one test done "for purpose of medical device", based on dossier information.

- 1 substance had one test with dossier information indicating it may have been for REACH and a second test that almost certainly was not performed for REACH. It was performed in China. This substance has an EFSA opinion for use as a food/feed additive, indicating it has an application outside cosmetic use.

- 5 substances were confirmed to have been tested for a purpose other than REACH; 3 of these were confirmed directly by the registrants and the other 2 were confirmed in the dossier.

- 1 substance had one test confirmed to be a test for China's regulation. A second test, a genotoxicity test, likely also was not performed for REACH, because the specific test is not required for REACH.

The 57 substances confirmed or likely tested for REACH have a total of 88 in vivo tests, 22 of which were directly confirmed by the registrants. These tests are further analyzed here by endpoint to understand the test approaches used for REACH and why an in vivo method may have been chosen over an available in vitro method.

\subsection{Skin and eye irritation}

Eleven substances had in vivo eye irritation tests, skin irritation tests, or both done after the Cosmetic Regulation deadline of March 2009 for these endpoints. Five of the substances had an in vivo eye irritation test only, three had an in vivo skin irritation test only, and three had both tests, for a total of 14 tests. No in vivo tests were performed after June 2016, when the REACH amendments required in vitro first.
Six of the 14 tests were confirmed by the registrants as tests for REACH. The other 8 tests may have been for REACH because the test years correspond to the REACH registration years, and 7 of them followed the REACH guidance of trying in vitro first.

Five tests were conducted in the stepwise approach recommended by REACH guidance. In this approach, the substance is first tested in vitro, and then the in vivo test is conducted only if the in vitro test gives equivocal results. Three dossiers directly state that equivocal in vitro results were the reason for the in vivo test. The dossiers for the other two substances contain no direct statements; however, both substances were tested per OECD TG 437 (BCOP), and for both the in vitro irritancy score (IVIS) was about 14 . This result indicated the test substance was not "corrosive" to eyes, but the testing strategy at that time (2011 and 2012) did not allow conclusions about "irritating to eyes" or "not requiring classification" for materials having an IVIS $\leq 55$, and so the in vivo test was required.

A sixth substance had a similar situation. The dossier states that the IVIS was well below 55, and the in vivo testing was then conducted for classification and labelling purposes in accordance with REACH guidance. The in vivo test had an unexpectedly severe outcome, which caused the registrant to question the in vitro results for the skin irritation test as well and to conduct a subsequent in vivo study for skin irritation.

For a seventh substance, the skin irritation test was combined with the acute dermal toxicity test for this Annex VIII substance. Annex VIII substances are required to have a second acute toxicity study in addition to the acute oral toxicity study. The second study for this substance was acute dermal toxicity, allowing the two tests to be combined.

For the remaining 4 substances, the dossiers gave no reason for the in vivo tests. Three of the substances, accounting for 3 eye irritation tests and 2 skin irritation tests, had the same registrant. The same registrant used in vitro eye and skin irritation methods for other substances registered under REACH. The fourth of those substances had only an in vivo eye irritation test.

\subsection{Acute toxicity}

Registrants responding to our inquiries confirmed eight of the acute toxicity tests performed after the March 2009 Cosmetic Regulation deadline were for REACH. Overall, REACH is the confirmed or likely reason for 36 acute oral and 5 acute dermal toxicity tests after the March 2009 Cosmetic Regulation deadline (Tab. 5). No acute inhalation toxicity studies were found.

The in vivo acute toxicity tests follow a pattern generally reflecting the REACH deadlines, peaking immediately before or at the 2010, 2013, and 2018 registration deadlines (Fig. 3).

In two cases, both dermal and oral tests were performed on the same substance. These substances are in Annex VIII (10100 tons/year), which requires two acute toxicity tests. The other three acute dermal toxicity tests, for substances in Annex VIII and Annex IX (100-1,000 tons/year), also are in addition to acute oral toxicity, but in those cases the acute oral toxicity endpoint was fulfilled by historical and read-across studies only.

The acute toxicity test is for hazard classification. This is one reason why acute toxicity information is often retrieved from 


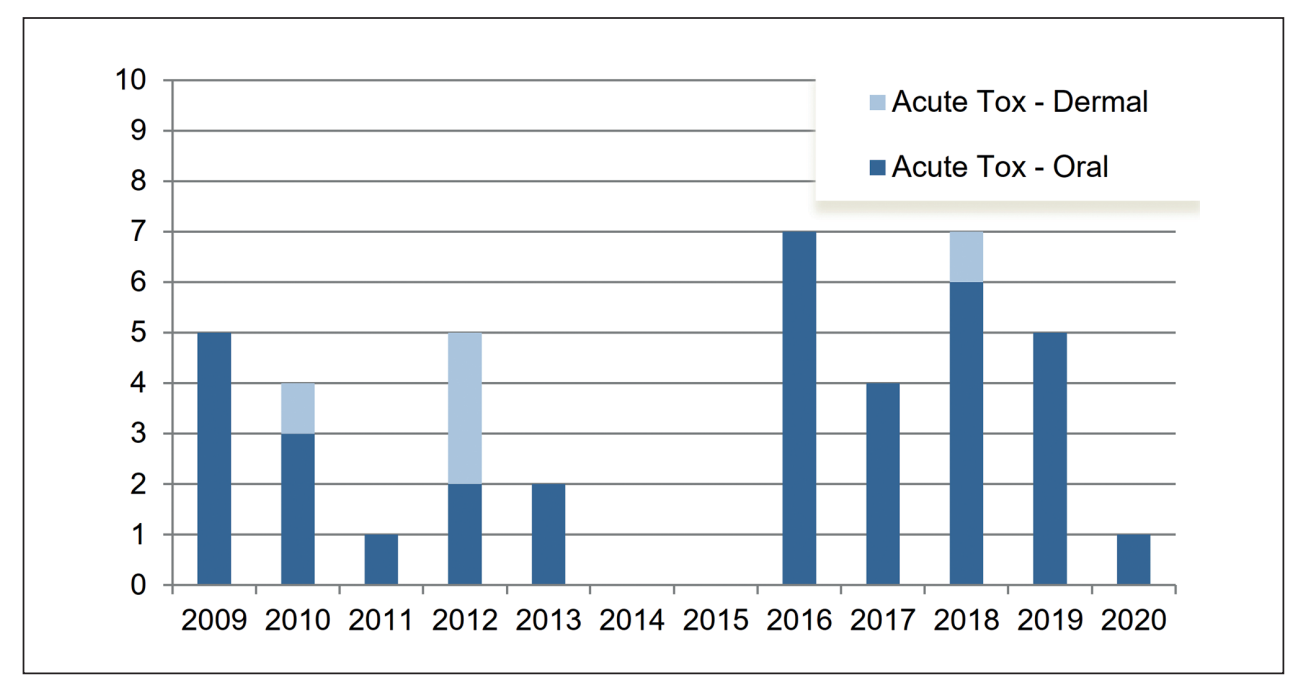

Fig. 3: Number of acute toxicity tests in REACH registration dossiers performed after the 11 March 2009 Cosmetic Regulation deadline These do not include the in vivo tests that were clearly not performed for REACH purposes. The trend follows the REACH registration deadlines of 2010 , 2013 and 2018.

other sources (Gissi et al., 2017) and also non-GLP data are accepted by regulators if sufficient data on the chemical identity are available. No in vitro tests are officially validated for acute toxicity. However, the ECHA guideline for information requirements indicates the in vitro NRU cytotoxicity assay is acceptable to justify the absence of hazard classification when combined with a QSAR prediction in a weight-of-evidence approach (ECHA, 2017).

Among the 36 substances that were tested in vivo for acute oral toxicity, only 5 have a classification for this hazard, indicating that in most cases the NRU/QSAR combination may have been sufficient for the hazard characterization. The dossiers do not indicate why the NRU/QSAR combination was not attempted. These may have been missed opportunities to avoid animal tests.

There were missed opportunities for waivers as well. Two of the 36 substances are classified H314, "Causes severe skin burns and eye damage", and both were tested for oral acute toxicity. REACH states in Annex VII that an oral toxicity study does "not generally need to be conducted if the substance is classified as corrosive to the skin". It is unknown if the skin irritation result was available before the in vivo acute toxicity test was performed as in both cases the skin irritation endpoint was evaluated with a weight-of-evidence approach using historical studies, and the dates of those analyses are not given.

Regulation EU 2016/863 amending REACH allows waivers of new in vivo tests for acute dermal toxicity after 2016 if the substance is not toxic by the oral route. Only one of the five dermal toxicity tests was on a substance that is classified H302, "Harmful if swallowed". Of the others, one was performed after 2016 and could therefore have been waived.

\subsection{Skin sensitization}

The Cosmetic Regulation deadline for ending in vivo skin sensitization tests was March 2013. Fifteen in vivo skin sensitization tests after this deadline likely were performed for REACH, based on test years that correspond to registration years and test approaches that match REACH guidance. Nine of the tests were done after October 2016, the effective date for the REACH requirement to use in vitro methods for this endpoint unless technically infeasible.

A review of the 15 dossiers provided the following details:

- Three substances were tested in vitro or used a QSAR study with positive results. One substance had all three in vitro tests for skin sensitization, with two positive results and one inconclusive result. A second substance also had all three in vitro tests for skin sensitization, with two negative and one positive result. The third substance's solubility was outside the domain of the in vitro tests, so a QSAR study was conducted to fulfil this endpoint requirement. The QSAR gave a positive prediction that could not be confirmed or rejected. In all cases, an in vivo local lymph node assay (LLNA) was then performed as required to classify the substance after the positive results. According to CLP, the distinction between category $1 \mathrm{~A}$ and $1 \mathrm{~B}$ for skin sensitization is possible only with the results from an in vivo test.

- Three substances had physical characteristics that made the in vitro methods infeasible. Two had low water solubility out of the applicability domain of the in vitro methods. For the third, the registrant notes that the available in vitro test methods were not applicable for this UVCB substance. These also used the LLNA method.

- Six other substances likely were out of the applicability domain of one or two of the three methods available at the time of the tests, based on the water solubility and partition coefficients of those substances.

- One substance had an in vivo LLNA documented after October 2016 that may have been an existing study. The dossier has a waiver request for the in vitro method, with the note: "Available study -1.1 . Use of existing data".

- No reason is given for not trying in vitro tests on the final two substances. These LLNA tests were done before the October 2016 deadline requiring use of in vitro tests first.

- Two of the 15 substances were tested using OECD TG 406, the guinea pig maximization test (GPMT), rather than the preferred LLNA. The GPMT should be avoided because the test 


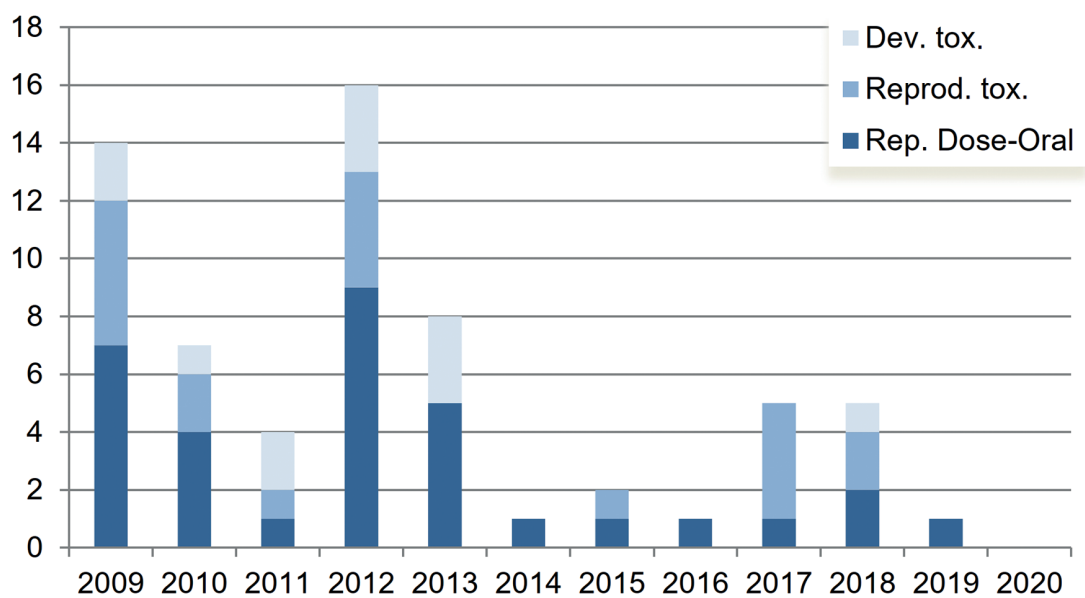

Fig. 4: Repeated dose, reproductive and developmental toxicity tests performed in the period 2009-2020 on cosmetic-only ingredients

Tests done after March 2013 exceed the Cosmetic Regulation deadline for repeated dose toxicity. In this chart, the tests listed under the bar for year 2013 were all performed before the March deadline except for two developmental toxicity tests. Tests using the combined method OECD TG 422 are included under Rep. Dose-Oral.

item is administered with an irritating agent, and the test is prolonged until the sensitization reaction occurs, causing more pain to the animals than the LLNA. The total number of animals is also higher. The GPMT is sometimes used if the substance is outside the applicability domain of the LLNA. The dossier for one of the substances states that the GPMT was in fact chosen because the test substance is a detergent, and surfactants are known to produce false-positive results in the LLNA. No reason is given for the use of the GPMT method for the other substance, a skin conditioning ingredient.

\subsection{Repeated dose toxicity, reproductive toxicity and developmental toxicity}

Compared to the previously analyzed endpoints, there are no defined non-animal strategies for the assessment of repeated dose toxicity. In the area of cosmetic ingredients, the only non-animal option is the use of read-across with similar substances (Rovida et al., 2020).

The Cosmetic Regulation deadline for repeated dose toxicity, reproductive toxicity and developmental toxicity was March 2013. Twelve substances were tested in one or more of these tests after the deadline, resulting in a total of 17 tests. Registrants confirmed that REACH was the reason for seven of the tests. The dossier for one substance confirmed that two other tests were performed for REACH. The other eight tests were likely conducted for REACH based on the test years, which coincide with the registration deadlines.

OECD TG 407 (28-day oral toxicity), 421 (screening reproductive toxicity), or 422 (combined repeated dose and reproductive toxicity) are test methods requested in REACH Annex VIII (substances in tonnage range 10-100 tons/year) but often also used in higher tonnage bands, for example, in support of a readacross strategy.

Ten of the 12 substances had one or more of these tests, of which one also had an OECD TG 408 (90-day oral toxicity, an Annex IX requirement) study:
- One substance: OECD TG 407 was the only Annex VIII repeated dose test.

- Three substances: OECD TG 421 was the only Annex VIII repeated dose test.

- Two substances: OECD TG 421 was performed with OECD TG 407.

- One substance: OECD TG 421 was performed with OECD TG 408 .

- Three substances: OECD TG 422 was performed.

Three of the substances are Annex IX substances. All three had an OECD TG 414 test, and one substance also had an OECD TG 408 test and an OECD TG 443 test. One OECD TG 414 test and the OECD TG 443 test were requested by ECHA as part of ECHA's evaluation process. Figure 4 shows the repeated dose tests from 2009 to 2020 . The match with REACH deadlines is apparent.

OECD TG 422 is the typical test done for an Annex VIII REACH registration dossier. The combination OECD TG $421+$ 407 is less common, because the possibility to perform the combined study with OECD TG 422 is fully accepted, more convenient, and requires fewer animals. The reason given for one OECD TG $421+407$ pair here was its higher statistical power, which increases the reliability of negative findings. Compared to OECD TG 407, both OECD TG 421 and 422 have only a screening status within ECHA. ECHA does not consider them for regulatory decisions regarding reproductive toxicity testing for substances registered according to REACH Annexes IX and X.

OECD TG 422 often is performed as a dose-finding study prior to higher tier tests such as OECD TG 443. Here, the OECD TG 443 test did have an OECD TG 422 test as a dose-finding study. Because that OECD TG 422 was used for dose-finding, it is not included in this analysis; however, such dose-finding studies also involve many animals. Three other repeated-dose tests done after the Cosmetic Regulation deadline were used as dose-finding studies, and also are not included here: two OECD 407 studies and one OECD TG 421 study. 


\subsection{Genotoxicity}

One in vivo genotoxicity test may have been done for REACH, based on test date and approach (Tab. 5). The substance was tested in vitro with positive results in a chromosomal aberration test and a test on gene mutation in mammalian cells. The in vivo test was negative, providing the opportunity to quit the classification need as mutagenic.

\subsection{Further verification}

Registrants and dossiers confirmed that some tests were done for a purpose other than REACH, such as for another industry use or regulation. These are reported in Section 4.2. Dossiers rarely report this information directly, however, so further analyses were done to evaluate possible non-REACH purposes.

\section{Dual uses in industries exempt from REACH registration}

Here, dual use refers to non-cosmetic uses that are exempt from REACH and so could not be screened out through the REACH database search described in Section 2.2 performed to identify cosmetic-only substances in the ECHA database. Such uses are as food/feed additives and as active substances for pharmaceutical, biocidal, and plant protection products. Most of the substances with in vivo tests after Cosmetic Regulation deadlines have only one registrant, so it is possible to review the business sectors of the registrant and evaluate potential dual uses in sectors exempt from REACH.

Registrants and dossiers confirmed the REACH or nonREACH purpose for tests on 21 substances, reported in Section 4.2 , so we consider just the remaining 42 substances here. The registrants of these substances are pharmaceutical or chemical companies, many of which serve exempt industries, or are "only representatives," which are firms hired to submit REACH dossiers on behalf of non-EU companies. Two registrants are confidential.

The potential for dual use in exempt areas was evaluated by searching the registrants' websites to identify business areas that are exempt from REACH. We also reviewed the dual use sections of SCCS and CIR opinions that were available for 11 of the substances. Finally, this information was considered against the test types, dates, and approaches.

The evaluation indicates that 27 of the 42 substances were more likely to have been tested only for REACH and not for a dual use. Each of these had two or more of the following factors indicating REACH as the more likely purpose:

- Test years coinciding with the REACH registration deadline: 20 substances, of which 18 are Annex VII or VIII substances tested in 2017-2018 and 2 are Annex IX substances tested in 2012-2013.

- Test years coinciding with the registration year of the substance: 27 substances.

- Registrants with no business areas exempt from REACH: 9 substances.
- Registrants whose only business area is cosmetics: 4 substances.

- Registrant website shows substance sold only as cosmetic ingredient: 5 substances.

- CIR/SCCS opinions state there are no identified dual uses for substance: 6 substances.

- CIR/SCCS opinions identify a dual use, but the use is not in the registrant's business area: 1 substance.

- REACH dossier states that the substance is used only in cosmetics: 2 substances.

- Tests are the specific REACH-required tests for that Annex: 2 substances, which were tested for the Annex VIII requirements for acute dermal toxicity and repeated dose-oral toxicity.

Two substances may have been tested for a dual purpose, based on the registrant business areas and the substance function. No conclusion could be drawn for the other 13 substances. Some had one of the characteristics in the preceding list, but this was not considered to be enough supporting information.

In conclusion, most companies that registered the cosmetic-only substances have interests in other areas, but the available evidence suggests that most tests were performed to comply with REACH.

\section{China's import and other regulatory requirements}

China has a list of allowed cosmetic ingredients, called the Inventory of Existing Cosmetic Ingredients in China (IECIC). Imported ingredients on this list require no new animal testing.

To determine if substances met this criterion, the substances were compared against the 2015 IECIC, which was the current list at the time of this paper. For 21 substances, the testing purpose is already known (Section 4.2). This left 42 substances to be checked against the 2015 IECIC list. ${ }^{10}$ Of the 42 substances, 16 were already on the 2015 IECIC list when they were tested, indicating those substances were not tested to obtain approval in China as a new ingredient.

To evaluate the remaining 26 substances, we looked at laboratory locations. Although tests for new imported ingredients theoretically were accepted from laboratories outside China, the tests usually were done in China. Laboratory locations were determined from the animal sources identified in the dossiers, because animals are usually bred near the testing laboratory. For 18 of the remaining 26 substances, the laboratory animal source is given and the source is outside China, making it unlikely those tests were performed to meet China's requirements. For the other 8 substances, no laboratory animal source is given.

From 2015 to early 2020, no new cosmetic ingredients were approved in China. This further supports the conclusion that China's import requirements do not account for the new animal tests. A substance could have undergone tests and then been disapproved but, as noted in the preceding paragraph, the laboratory animal sources indicate this is unlikely.

\footnotetext{
10 An official website for IECIC is: http://www.pharmacovigilance.org.cn/art/2020/12/3/art 165586 10085661.html. This site is not available outside China or in English. Third-party sites available in English: https://www.chemsafetypro.com/Topics/Cosmetics/China_IECIC_Finder.html (accessed 05.02.2021), and http://www.cirs-reach.com/ news/SFDA_Consults_On_Draft_Third_Batch_of_IECIC_2012.html (accessed 05.02.2021).)
} 
In December 2020, four new ingredients were approved as new cosmetic raw materials ${ }^{11}$ in China; however, none of these contributed to the in vivo tests reported for cosmetic-only substances after the Cosmetic Regulation deadlines.

In April 2021, the Chinese National Medical Products Administration (NMPA) released a new regulation called "Guideline on cosmetics efficacy claim evaluation" 12 , which took effect 1 May 2021, stating that most cosmetic efficacy claims require a human test, consumer research, or laboratory testing to prove the claim. The laboratory testing includes in vivo and in vitro tests on the active ingredient, but they are optional and can be replaced by a human test or consumer trial research (in China, animal tests are mandatory before a human test can be done). This regulation cannot account for the new tests because the tests identified in this study all were conducted before November 2020.

Finally, in January 2021, the NMPA issued a new draft regulation called "Toothpaste Filing Documents specification"13, which mentions that toothpaste shall be regulated as general cosmetics. NMPA is now working on the approved list for toothpaste filings, and some toothpaste ingredients will be required to have an acute oral toxicity test. However, the regulation is new, and all tests reported for this paper were conducted before the draft regulation was issued.

Based on these data, China's import requirements and other regulatory requirements are unlikely to be a significant reason for in vivo tests on cosmetic ingredients performed after the Cosmetic Regulation deadlines and reported in this study.

\section{Requirements of US, Japan, and other countries}

Two substances are reported to have been tested for countries outside the EU and China in the dossiers. These two substances account for seven tests. For other substances, the possibility that tests were conducted for regulations in other countries was evaluated indirectly.

Other countries generally do not have an approved existing ingredient list similar to China's IECIC. Possible import-related tests for other countries, therefore, were evaluated by reviewing the cosmetic function of each substance. Countries generally have categories of restricted use ingredients for which safety testing could be required for new ingredients. Each country has its own list of such ingredients, but they commonly include sunscreen, preservative, antiperspirant, oral care, dandruff, and bleaching (skin whitening) ingredients. Of the 63 substances with in vivo tests done after Cosmetic Regulation deadlines, cosmetic functions were identified for 53 substances. Ten of these substances have a function that could fall into a country's restricted category. These 10 substances have a total of 15 tests done after Cosmetic Regulation deadlines. The substances with unknown function also have a total of 15 tests done after Cosmetic Regulation deadlines. Three of these tests are known to have been done for REACH, as confirmed by the registrant, leaving 12 tests to consider. Combined, therefore, there are $27(15+12)$ tests that have greater or unknown potential for being conducted for a non-EU country's regulations. These 27 tests represent $26 \%$ of the total tests for human health endpoints done after the Cosmetic Regulation deadlines.

Brexit is an issue, but at the moment the situation is still not clear on whether the testing ban on cosmetic ingredients and products will be implemented also in the UK and how the UK REACH will deal with it. Our analysis was performed before Brexit went into effect.

The evaluations are indirect, but they indicate that import or other safety requirements of non-EU countries likely do not account for most in vivo tests on cosmetic-only substances reported in REACH after the Cosmetic Regulation deadlines.

\section{Ongoing and future in vivo tests}

The dossiers for cosmetic substances undergo the same procedures as all other REACH registration dossiers.

One of these is the testing proposal procedure triggered when the registration dossier lacks information required for Annex IX (tonnage band 100-1,000 tons/year) or Annex X (tonnage band $>1,000$ tons/year). In that case, according to Article 22(h) of $\mathrm{REACH}$, the tests cannot be performed immediately; rather, the registrant must submit a testing proposal and wait for formal approval from ECHA. All details are reported on the ECHA website ${ }^{14}$.

Another procedure is the evaluation process that is either a completeness check of the dossier performed manually by ECHA when the dossier is submitted or updated, or a review initiated by a Member State within the activity of the Member State Committee (MSC). Substances that are under evaluation are published on the ECHA website and listed on the Community Rolling Action Plan (CoRAP) ${ }^{15}$. Based on our analysis performed in March 2021, of the 413 unique cosmetic-only substances, 6 are on the list for testing proposals for one or more studies and 13 are on the CoRAP list (one substance is on both lists).

The ECHA decisions on testing proposals approved the OECD TG 408 (90-day repeated dose toxicity) study identified in Section 4.6. Two other testing proposals contain requests related to OECD TG 416 and one other to OECD TG 408 and 414, but there is no evidence of those tests in the corresponding dossiers. One testing proposal did not contain a request for in vivo tests, and another is also on the CoRAP list, and it is not possible to understand what the initial testing proposal contained.

The situation of the substances on the CoRAP list is more complex. The analyses of the 13 dossiers of cosmetic-only substances revealed that one decision pertained only to environmental

\footnotetext{
11 https://www.nmpa.gov.cn/xxgk/ggtg/qtggtg/20201228172412190.html

12 https://www.nmpa.gov.cn/xxgk/zhqyj/zhqyjhzhp/20201105145412189.html

13 https://www.nmpa.gov.cn/xxgk/zhqyj/zhqyjhzhp/20210106145013118.html

$14 \mathrm{https}: / /$ echa.europa.eu/information-on-chemicals/testing-proposals

15 https://echa.europa.eu/information-on-chemicals/evaluation/community-rolling-action-plan/corap-table
} 
tests. Two decisions are not public, and the corresponding dossiers contained no in vivo tests performed after 2009 in one case, while the other one has an OECD TG 416 but with no indication of the period when it was done, so it was not possible to conclude whether it was performed after 2013.

One substance has two ECHA decisions, one asking for OECD TG 408 and 414 and one asking for OECD TG 443. All three tests are now present in the dossier. This substance is also included on the list for endocrine assessment. According to REACH, endocrine activity is considered as a condition to enter into the list of SVHC substances and to prohibit the use as biocide ingredient. For this reason, ECHA established a group of experts dedicated to the identification of substances that may have this property in $2013^{16}$. At the time of our assessment (March 2021), this list contained 98 substances, 5 of which are cosmetic-only substances. These assessments for endocrine disruptor activity are still ongoing, and it is not clear if ECHA will decide based on the available information only or will require additional tests.

All remaining decisions for cosmetic-only ingredients on the CoRAP list have a deadline for updating the correspondent dossier that has not yet expired, and therefore the new in vivo tests will be published in the future. In total, without considering environmental tests, they contain requests for the following additional tests:

- 7 tests according to OECD TG 408

- 7 tests according to OECD TG 414

- 3 tests according to OECD TG 422

- 3 tests according to OECD TG 443

The ECHA decisions contain detailed explanations about why the submitted dossiers were considered noncompliant. A common reason for rejecting the initial dossier is the unsuitability of the existing studies, sometimes because they have no GLP certificate, because they have less data compared to the OECD guideline that is used now, or because the highest tested dose did not cause toxicity to the animals, as required in the OECD test guidelines. In the case of read-across, ECHA usually mentions the lack of a strong justification for the similarity between source and target substances. A good opportunity to overcome this limitation is through the performance of suitable in vitro tests on both source and target substances to compare their biological activity with the possibility to discuss whether any difference can represent a risk in the toxicological assessment of the substance (Rovida et al., 2020, 2021).

The registrant has the right to appeal the ECHA decision, and this is what happened with the case described in the introduction. In another appeal for a different cosmetic-only substance, the submitter claimed the cosmetic ingredient status of the substance for not accepting the ECHA decision to perform new in vivo tests, in that case OECD TG 408 and 422. The board of appeal annulled the initial ECHA decision, but ECHA presented it again, explaining that the board of appeal only contested the lack of explanation on the relationship between REACH and the Cosmetic Regulation. The second decision was based on the state- ment that "registrants of substances that are exclusively used in cosmetics may not perform animal testing to meet the information requirements of the REACH human health endpoints unless such tests are needed to assess the risks from exposure to workers". The registrant now must submit the data by a deadline.

ECHA currently is reviewing dossiers from the 2010 and 2013 REACH deadlines (substances $\geq 100$ tons/year), which apply to about $15 \%$ of the cosmetic-only ingredients analyzed in this paper. Most ( $85 \%$ ) fall under the 2018 REACH deadline (substances $<100$ tons/year), which ECHA plans to review by 2027 , so those reviews and potential requests for additional studies are still pending. ${ }^{17}$

In conclusion, the number of new in vivo tests performed on cosmetic substances will increase in the future. ECHA asks for new in vivo tests as soon as the evaluation of the alternative strategy, mainly read-across, or the justification for waiving the new tests are deemed unsatisfactory. In theory, the registrant can still update the dossier by adjusting the justifications instead of doing a new in vivo test. However, the risk for the registrants is high, because if the adaptation to the standard information requirement is rejected a second time, the ECHA decision can lead to the revocation of the registration number and the prohibition of marketing the substance.

\section{Conclusions}

The results of this analysis give cause for both optimism and concern. The review of the dossiers of cosmetic-only ingredients shows in vivo testing declined steeply after 2009, when the initial cosmetic testing ban took effect. However, the testing did not end then or in 2013 when the final ban came into effect. The trends show continued in vivo testing of cosmetic-only ingredients for REACH, and this is likely to carry on as ECHA continues to evaluate REACH registration dossiers.

The REACH database contained 3,206 chemical dossiers with cosmetics as a reported use at the time of our assessment in December 2020. Of these dossiers, 419 reported cosmetics as the only use, and 63 of these had in vivo tests that were done after the Cosmetic Regulation ban. For dossiers with in vivo tests, most $(88 \%)$ were historical studies pre-dating 2009 . About $20 \%$ of dossiers avoided in vivo testing for all human health endpoints by using alternative methods, indicating that it is possible to use only alternative methods. Many other dossiers used exclusively alternative methods for at least some endpoints.

The new in vivo tests were largely performed because they were required by REACH. For health endpoints with available in vitro methods, most registrants who reported in vivo tests had followed the REACH principle of in vitro first, but ultimately had to test in vivo to comply with REACH. Key reasons were positive or equivocal results from in vitro tests or chemical properties that made in vitro tests infeasible. Some of these tests could have been waived by applying the possibilities listed in REACH

\footnotetext{
16 https://echa.europa.eu/ed-assessment

17 https://echa.europa.eu/dossier-evaluation
} 
Annex XI. In particular, the dossiers include many new in vivo tests for acute toxicity, despite opportunities to use strategies not involving animals.

More new in vivo testing for REACH is likely. As part of its dossier review process to date, ECHA has already requested new in vivo tests for cosmetic-only substances, and more requests can be expected as ECHA identifies data gaps in the dossiers. Also, ECHA's decision that in vivo testing may be performed on cosmetic-only ingredients "to assess the risks from exposure to workers" affects most ingredients. Except for import of a finished cosmetic product, all other cosmetic processes involve worker exposure to the cosmetic ingredient.

In vivo testing of cosmetic-only ingredients for REACH has not previously come to attention because the EU no longer has a method of tracking in vivo tests on cosmetic ingredients. The EU's status report on animal use counts all REACH tests, including on cosmetic ingredients, as "industrial chemicals legislation" tests. The most recent report, in 2020, states no testing was reported for the Cosmetic Regulation, but REACH records make clear that testing of cosmetic ingredients continues under REACH. Identifying in vivo tests on cosmetic substances in REACH would allow this important metric to be tracked again.

The conflict between REACH and the Cosmetic Regulation poses a serious dilemma for all segments of the cosmetic industry: for ingredient manufacturers, as they can be legally required under REACH to conduct in vivo tests on their ingredients, but the cosmetic market may reject ingredients with such tests; for cosmetic brands, as they cannot easily identify REACH testing of ingredients in their supply chain, but if such testing is identified, a brand risks backlash from consumers if it continues to use the ingredient, but finding an alternative can be difficult and costly; and for consumers, as they can no longer have confidence that the EU cosmetic products they purchase were not tested on animals. Greater transparency on the post-ban in vivo testing and an engaged effort by stakeholders to resolve the conflict would support the cosmetic industry's effort to be free of animal testing and help retain consumer confidence in their products.

\section{References}

Adler, S., Basketter, D., Creton, S. et al. (2011). Alternative (non-animal) methods for cosmetics testing: Current status and future prospects - 2010. Arch Toxicol 85, 367-485. doi:10.1007/s00204-011-0693-2

Busquet, F., Kleensang, A., Rovida, C. et al. (2020). New European Union statistics on laboratory animal use - What really counts! ALTEX 37, 167-186. doi:10.14573/altex.2003241

Chesnut, M., Yamada, T., Adams, T. et al. (2018). Regulatory acceptance of read-across: Report from an international satellite meeting at the $56^{\text {th }}$ annual meeting of the society of toxicology. ALTEX 35, 413-419. doi:10.14573/altex.1805081

EC (2009). Regulation (EC) No 1223/2009 of the European Parliament and of the Council of 30 November 2009 on Cosmetic Products. OJ L342, 64. doi:10.3000/17252555.L_2009.342. eng
ECHA (2017). Guidance on Information Requirements and Chemical Safety Assessment Chapter R.7a: Endpoint specific guidance. ECHA-17-G-18-EN. doi:10.2823/337352

ECHA (2020). The Use of Alternatives to Testing on Animals for the REACH Regulation. Fourth report under Article 117(3) of the REACH Regulation. June 2020. ECHA-20-R-08-EN. doi:10.2823/092305

Gissi, A., Louekari, K., Hoffstadt, L. et al. (2017). Alternative acute oral toxicity assessment under REACH based on subacute toxicity values. ALTEX 34, 353-361. doi:10.14573/altex. 1609121

Hartung, T. (2008). Food for thought ... on alternative methods for cosmetics safety testing. ALTEX 25, 147-162. doi:10.14573/ altex.2008.3.147

Hartung, T. and Rovida, C. (2009). Chemical regulators have overreached. Nature 460, 1080-1081. doi:10.1038/4601080a

Hartung, T. (2010). Food for thought ... on alternative methods for chemical safety testing. ALTEX 27, 3-14. doi:10.14573/ altex.2010.1.3

Linkov, I., Massey, O., Keisler, J. et al. (2015). From “weight of evidence" to quantitative data integration using multicriteria decision analysis and Bayesian methods. ALTEX 32, 3-8. doi:10.14573/altex.1412231

Patlewicz, G., Ball, N., Becker, R. A. et al. (2014). Read-across approaches - Misconceptions, promises and challenges ahead. ALTEX 31, 87-396. doi:10.14573/altex.1410071

Rovida, C. and Hartung, T. (2009). Re-evaluation of animal numbers and costs for in vivo tests to accomplish REACH legislation requirements for chemicals - A report by the transatlantic think tank for toxicology (t $\left.{ }^{4}\right)$. ALTEX 26, 187-208. doi:10.14573/altex.2009.3.187

Rovida, C., Barton-Maclaren, T., Benfenati, E. et al. (2020). Internationalisation of read-across as a validated new approach method (NAM) for regulatory toxicology. ALTEX 37, 579606. doi:10.14573/altex.1912181

Rovida, C., Escher, S. E., Herzler, M. et al. (2021). NAM-suph ported read-across: From case studies to regulatory guidance in safety assessment. ALTEX 38, 140-150. doi:10.14573/ altex.2010062

\section{Conflict of interest}

The authors declare that they have no conflicts of interest.

\section{Acknowledgments}

This work was supported by the 3 Rs center program (NAMACCEPT) of the Baden-Wuerttemberg Ministry for Science, Research and Art (MWK Baden-Württemberg) and the Doerenkamp-Zbinden Foundation. We are very grateful to the REACH registrants who provided further information on the reasons why in vivo tests were performed in their dossiers. We are also deeply grateful to the peer reviewers for their patient review and thoughtful comments. 